\title{
1 Genomic variation in the tea leafhopper reveals the basis of 2 adaptive evolution
}

3 Qian Zhao ${ }^{1, \#}$, Longqing Shi ${ }^{1,2, \#}$, Weiyi $\mathrm{He}^{1}$, Jinyu $\mathrm{Li}^{1,3}$, Shijun $\mathrm{You}^{1}$, Shuai Chen ${ }^{4}$, Jing

$4 \quad$ Lin $^{4}$, Yibin Wang ${ }^{4}$, Liwen Zhang ${ }^{1}$, Guang Yang ${ }^{1}$, Liette Vasseur ${ }^{1,5}$, Minsheng You ${ }^{1 *}$

5

$6{ }^{1}$ State Key Laboratory of Ecological Pest Control for Fujian and Taiwan Crops, 7 Institute of Applied Ecology, Fujian Agriculture and Forestry University, Fuzhou 8 350002, China

$9{ }^{2}$ Institute of Rice, Fujian Academy of Agricultural Sciences, Fuzhou 350018, China

$10{ }^{3}$ Tea Research Institute, Fujian Academy of Agricultural Sciences, Fuzhou 350001, 11 China

$12{ }^{4}$ Center for Genomics and Biotechnology, Fujian Provincial Key Laboratory of 13 Haixia Applied Plant Systems Biology, Key Laboratory of Genetics, Fujian Agriculture and Forestry University, Fuzhou 350002, China

${ }^{5}$ Department of Biological Sciences, Brock University, 1812 Sir Isaac Brock Way, St. Catharines, ON L2S 3A1, Canada

17 \#These authors contributed equally to this work: Qian Zhao and Longqing Shi.

*Correspondence should be addressed to Minsheng You (msyou@ fafu.edu.cn,

ORCID \#0000-0001-9042-6432)

Running title: Zhao Q et al/ Adaptive evolution of Empoasca onukii

Counts of words: 5751

Tables and figures : 4 tables and 4 figures 


\section{ABSTRACT}

The tea green leafhopper (TGL), Empoasca onukii, is of biological and economic interest. Despite numerous studies, the mechanisms underlying its adaptation and evolution remain enigmatic. Here, we used previously untapped genome and population genetics approaches to examine how this pest so rapidly has adapted to different environmental variables and thus has expanded geographically. We complete a chromosome-level assembly and annotation of the E. onukii genome, showing notable expansions of gene families associated with adaptation to chemoreception and detoxification. Genomic signals indicating balancing selection highlight metabolic pathways involved in adaptation to a wide range of tea varieties grown across ecologically diverse regions. Patterns of genetic variation among 54 E. onukii samples unveil the population structure and evolutionary history across different tea-growing regions in China. Our results demonstrate that the genomic change in key pathways, including those linked to metabolism, circadian rhythms and immune system function, may underlie the successful spread and adaptation of E. onukii. This work highlights the genetic and molecular bases underlying the evolutionary success of a species with broad economic impact, and provides insight into insect adaptation to host plants, which will ultimately facilitate more sustainable pest management.

KEYWORDS: tea green leafhopper; genomic variation; population genetics; local adaptation; evolutionary history 


\section{Introduction}

Tea is the most popular beverage worldwide, surpassing coffee and cocoa, with a production of 6.1 million metric tons in 2019 (ITC; https://www.statista.com/statistics/264183/global-production-and-exports-of-tea-since-2004/).

China represents the largest tea producer, consumer, and exporter in the world. In Asia, the tea green leafhopper (TGL), Empoasca onukii (Hemiptera: Cicadellidae), represents the most devastating pest across tea plantations, causing up to 50\% economic loss of tea production annually $[1,2]$. Both nymph and adult TGLs pierce and suck the sap of tender tea shoots, which are the most important part of the plant to produce high-quality tea. Adult females also lay their eggs in these shoots, leading to irreparable damage (Figure S1) [2, 3]. Presence of local TGL populations has been recorded in China since the 1950s [4]. Its distribution has increased around tea-producing regions of China, Japan, and Vietnam [5]. E. onukii can cause yield loss of between 15 and $50 \%$, up to $100 \%$ in severely damaged plantations [2, 6].

E .onukii belongs to the most species-rich hemimetabolous order, various species of which are agricultural pests or human disease vectors [7]. As a monophagous insect, TGL is well-adapted, both physiologically and biochemically, to different tea varieties [8]. Thus, the rapid expansion of E. onukii raises critical questions concerning which factors contribute to its successful dispersal and colonization, and how genomic architecture underlies its broad and rapid ability to adapt.

To address the above questions, we generated a chromosomal level genome assembly of the E. onukii by integrating Illumina short reads, Oxford Nanopore long reads, and high-throughput chromosome conformation capture (Hi-C technology). This high-quality genome resource enabled us to investigate the genetic basis of chemoreception and detoxification in this insect, key to adapting to new environments. Based on 54 re-sequenced genomes of the E. onukii samples collected from different locations across a diverse range of tea-growing regions in China, we analyzed patterns 
of genomic variation and population structure in this species, allowing us to gain insights into its evolutionary history and successful, rapid spread and colonization.

\section{Results and Discussion}

\section{Chromosome-level assembly of the tea green leafhopper}

The genome of E. onukii was estimated to be $\sim 608 \mathrm{Mb}$ based on K-mer analysis. We combined 61× Illumina short read and 109x Nanopore ONT sequences with chromosome-scale scaffolding. We informed our assembly using physical mapping of high-throughput chromatin conformation capture (Hi-C) (Tables S1 and S2), to generate an assembly based on $599 \mathrm{Mb}$ of sequence, with the mitochondrial sequences excluded (Table 1 and Tables S3, S4). This assembly accounted for $98.5 \%$ of the estimated genome size. A total of $592 \mathrm{Mb}$ sequences and $98.83 \%$ of assembled sequences were then anchored onto 10 pseudo-chromosomes using ALLHiC (see Methods and Tables 1 and 2, Figures 1A and S2A). An official gene set was generated based on alignment of insect gene homologs, ab initio predictions, and transcriptomic evidence. Genome annotation predicted 19,642 protein coding genes with $92.5 \%$ BUSCO completeness in TGLs (Table 1 and S5). The sequenced E. onukii genome showed high heterozygosity (2.8\%), with 13,122,207 heterozygous SNPs, 3,796,369 heterozygous indels, and complex segmental duplication patterns (Figure 1A). We also assembled the mitochondrial genome, which had a total length of $14.2 \mathrm{~kb}$ and 13 protein-coding genes annotated (Figure S2B).

Compared to recently published Hemiptera genomes $[9,10]$, this assembly is a high-quality genome with 92.7\% BUSCO completeness (Tables S4 and S6). Around $92.3 \%$ (337.5/365.8 million) of the Illumina short reads were mapped to the assembled reference, representing about $\sim 94 \%$ of the genome (Table S7). Well-organized patterns of interacting contact along the diagonal for each pseudo-chromosome confirmed the high-quality chromosome-level assembly (Figure S2). In addition, assessment using the LTR Assembly Index (LAI) [11] revealed that more intact LTRs were recalled in our assembly than previously published insect 
103

104

105

106

107

108

109

110

111

112

113

114

115

116

117

118

119

120

121

122

123

124

125

126

127

128

129

130

131

genomes $[9,10]$, further supporting the high quality of the TGL genome (Figure S3A).

In total, 19,642 genes were annotated in E. onukii and compared with five other well-annotated Hemiptera published genomes including Diuraphis noxia [12], Acyrthosiphon pisum [13], Cimex lectularius [9], Nilaparvata lugens [14], and Myzus persicae (https://bipaa.genouest.org/sp/acyrthosiphon_pisum/). Results showed that $56.9 \%(11,170 / 19,642)$ of E. onukii genes had homologs (Figure 1B). The E. onukii genome contained $\sim 37.7 \%$ repetitive sequences, a relatively moderate level among published Hemiptera genomes, which repetitive sequences range from $\sim 12 \%$ in $D$. noxia [12] to $56.5 \%$ in C. lectularius [9]. TEs accounted for approximately $32.8 \%$ of the E. onukii genome, and were comprised of $12.82 \%$ transposon sequences, $4 \%$ long terminal repeats (LTRs), 11.24\% long interspersed nuclear elements (LINEs), 2.20\% short interspersed nuclear elements (SINEs) (Table S8). TE level in E. onukii was comparable to that of $N$. lugens [15], but approximately 1.5 times higher than $A$. glycines [10] (Figure 1C). Similar to the aphid genome, LINEs were more prevalent than LTR retroelements (Table S8). The moderate genome size and levels of repetitive sequences in E. onukii compared to other hemipteran species (Figure 1C; Figure S3B) indicated that TEs and other non-coding DNA might contribute to variation in genome size [16]. The genome sizes of insect generally result from changes in the repetitive DNA content [16]. Therefore, we calculated the correlation between repetitive sequence content and genome size in sequenced insect species from both the Holometabola (e.g., flies, beetles, wasps, and butterflies) and Hemimetabola (e.g., aphids, true bugs, blood-feeding bugs, and leafhoppers). As expected, the genome size was highly correlated with the DNA repetitive content (Spearman test, $r=0.8, P=$ 0.0004) (Figure S3B). Previous studies suggest that differences in DNA repeat content are likely due to TE variation or the influence of stochastic population effects [17].

We used OrthoFinder to identify orthologous genes across the genomes of $E$. onukii and other 18 insect species covering six different insect orders (Hemiptera, Isoptera, Hymenoptera, Coleoptera, Diptera and Lepidoptera). A total of 196 
single-copy orthologous genes, 6411 multi-copied orthologous genes, 18 unique paralogous genes and 3325 unclustered genes were identified. The phylogenetic relationships among 19 sequenced insect species were analyzed using the PROTGAMMALGX model in RAxML [18] based on the 196 single-copy orthologous genes (Figure 1C). Based on these analyses, E. onukii was estimated to have diverged from $N$. lugens and L. striatellus approximately 175 Mya ago (Figure S4).

Expansion and contraction of gene families were analyzed based on 19 species. Results showed that both total and species-specific genes in Hemiptera genomes increased relative to other insect orders (Figure S4) [15]. We identified 2,859 novel genes (species-specific) in E. onukii, representing about $14.5 \%$ of the genome. In addition, 1178 expanded gene families were detected and these gene families were over-represented in specific Gene Ontology (GO) terms, including carboxylic ester hydrolase activity, zinc ion binding, iron ion binding, and transmembrane transporter activity (Table S9). The E. onukii genome contained 3880 contracted genes families (Figure S4). For example, functional analysis revealed that these genes were involved in immunity (immunoglobulins), myosin, and tropomyosin (Table S10). These E. onukii-specific gene family expansions and contractions were likely involved in evolutionary adaption to tea phloem sap, symbiotic dependence, pathogen immunity, and environmental conditions such as ecological and climatic variation, and tea variety differences. For example, evidence shows carboxylic ester hydrolase activity is involved in sap-sucking insects (e.g., M. persicae and S. graminum) sequestering OP insecticides [19]. Immunoglobulin superfamily proteins have been reported as candidates for synapse targeting functions related to synaptic specificity in the visual system in Drosophila [20, 21].

\section{Genomic adaptation to chemoreception and detoxification}

The chemosensory system is essential for herbivorous insects to orient toward and locate potential host plants [22], potentially indicating how herbivorous insects adapt to host changes. Environmental signals and chemosensory stimuli are 
recognized and transduced by several multi-gene families including olfactory receptors (ORs), ionotropic receptors (IRs), gustatory receptors (GRs), odorant-binding proteins (OBPs), and chemosensory proteins (CSPs) [22, 23]. To examine genes linked to chemosensory stimuli recognition, we manually annotated several related gene families, including 20 olfactory receptors (ORs), 23 ionotropic receptors (IRs), 12 gustatory receptors (GRs), 5 odorant-binding proteins (OBPs), and 26 chemosensory proteins (CSPs) (Table 3).

Comparative analysis of genomes across different species revealed an increased number of CSPs in the E. onukii genome (Table 3, Figure 2A and B, and Table S11). The phylogenetic analysis of Hemiptera identified 10 homologous subgroups of CSPs (CSP1-CSP10) (Figure 2B), which was consistent with a previous study [24]. Other than CSP5 and CSP6, E. onukii CSPs were present in seven of the ten clades, indicating these genes are highly conserved across the Hemiptera. Interestingly, we found obvious expansion of some subgroups in E. onukii (e.g., CSP3, CSP4, CSP8, and CSP9) and these CSPs were unevenly distributed over 4 of the 10 chromosomes, with enrichment on chromosome 1 (Fisher exact test, $P$ value $<0.00001$; Figure $2 \mathrm{C}$ ). Most CSP genes were distributed in expanded clusters on chromosomes, likely through a series of gene duplication events (Figure 2C). Meanwhile, several CSPs were highly expressed across different life cycle stages (Figure S5A), implying an important role in the growth and development of E. onukii. Earlier studies on CSP functions [25, 26], coupled with our observations of conserved phylogeny in Hemiptera species and species-specific expansion of CSPs, indicate that CSPs are crucial for recognition of tea volatiles and location of potential host tea plants. We suggest that E. onukii requires many CSPs to specifically detect the complex molecular components of odors from different tea cultivars. Thus, our analyses highlighted directions for further experimental analysis of genes linked to host adaptation. Toward this goal, functional testing of CSPs might identify genes that were responsible for detection of specific tea cultivars by E. onukii. 
Our investigation of the chemoreceptor-related genes showed relatively low numbers of ORs, IRs, GRs and OBPs in E. onukii (Table 3; Table S11; Figure S5-8). For example, we found that the number of OBPs in Hemiptera species was lower than other insect orders (Figure S5B), suggesting conservation of odorant molecular transport in Hemiptera [27]. Other chemoreceptor genes, including ORs, GRs and IRs, play important roles in local adaption by responding to chemical signals with neuronal activity [15]. The species-specific expansion OR clade of gene family was obvious in our analysis (Figure S6). Polyphagous insects (e.g., P. americana) possess more OR genes than monophagous insects (e.g., E. onukii) (Table 3; Table S11; Figure S6). This might have resulted from specific evolutionary adaption to food selection and detection since genetic diversity of ORs allows insects to bind to a greater range of ligands [28]. In addition, similar to N. lugens, E. onukii had a substantially lower number of GRs (Table 3, Figure S7). Earlier studies show a close relationship between GRs and insect herbivory, with lower number of GRs in specialists than in generalists $[29,30]$. Another explanation may be that antennae of leafhoppers have a much simpler structure with fewer sensilla than those of planthoppers (e.g., N. lugens) and aphids. TGL also possessed fewer IRs (Table 3; Table S11; Figure S8), which mediate synaptic communication in insects and mediates responses to volatile chemicals in D. melanogaster $[31,32]$. We believed that the numerical reduction in ORs, IRs, GRs and OBPs might be associated with the adaptive evolution to a monophagous diet of tea phloem sap, and the substantial expansion of CSPs might contribute to tea volatile perception in E. onukii.

E. onukii is believed to have experienced rapid evolution leading to insecticide resistance in natural populations [33]. Four classic gene families commonly associated with detoxification of xenobiotics and insecticides, including P450, COEs, GSTs and ABCs, were therefore investigated. We identified 103 cytochrome P450s, 29 ATP-binding cassette transporters (ABC transporters), 77 carboxylesterases (COEs), and 30 glutathione S-transferases (GSTs) (Table 3). Similar to other insecticide resistant pests [34], we found that the P450 gene family was expanded, 
mainly in CYP3 and CYP4 clans (Table 3; Table S12; Figure 2D; Figure S9A). Based on our RNA-seq data, 28 CYP3 and 38 CYP4 genes showed expression (FPKM > 1.0) with 20 in CYP3 and 16 in CYP4 being highly expressed during at least one developmental stage $($ FPKM > 10.0) (Figure 2D; Figure S9B). The results underlined their potential function of detoxifying the xenobiotics or insecticides in TGL.

We tracked the expression patterns of these genes in TGL samples collected from different tea cultivars, with four cultivars being resistant and four cultivars susceptible to TGL according to previous studies [35]. Results showed that 17 CYP3 and 12 CYP4 genes were highly expressed $\left(\log _{2}{ }^{\text {FRM }}>10.0\right)$ but not differentiated in both resistant and susceptible tea cultivars (Figure 2D; Figure S9C). Thus, we speculated that CYP genes might be involved in metabolism of common xenobiotics, or their expression may be induced by insecticides. Indeed, previous studies have shown that CYP3s are involved in xenobiotic metabolism and insecticide resistance, with some family members being inducible by pesticides or plant secondary metabolites [36]. CYP4s are known to encode constitutive and inducible enzymes related to odorant and pheromone metabolism, and expression can be induced by xenobiotics [37].

Niche under adaptive selection are related to metabolic regulation and detoxification

E. onukii samples were collected from four tea-growing regions around China: southwest region (SWR), south of the Yangtze River region (SYR), north of the Yangtze River region (NYR), and south China region (SER), and these samples were re-sequenced with a depth ranging from $20.8 \times$ to $30.7 \times$ at whole genome level (Table S13). After filtering the low-quality variants, we generated a genomic dataset containing 12,271,501 high-quality SNPs (Table S13) to estimate the genomic signatures of evolutionary adaptation for E. onukii, based on Tajima's $D$ with a $50-\mathrm{kb}$ window size and a fixed step length of $10 \mathrm{~kb}$. Totally 369 sliding windows, covering 18.45 $\mathrm{Mb}$ genomic sequences (Table S14) and containing 82 protein-coding genes (Table S15) were detected, including seven genes (Table S16) under purifying 
Purifying selection is important in shaping genomic diversity in natural populations and is essential to preserving biological functions at selection sites [38]. Almost all these genes are related to nervous system or visual functions. For instance, forkhead box protein $\mathrm{P} 1(F O X P 1)$ is a transcription factor with a regulatory function in the central nervous system (CNS), and mutations in this gene have been linked to various neurodevelopmental diseases, including autism, cognitive abnormalities, intellectual disabilities and speech defects [39]. Coronin 6 is highly enriched at the adult neuromuscular junction and can regulate acetylcholine receptor (neurotransmitter receptor) clusters by modulating interactions between the actin cytoskeletal network and receptors [40]. In mice lacking SZT2, mTORC1 signaling is hyperactive in several tissues including neurons in the brain, and these components have been linked to neurological disease [17]. The nucleoredoxin-like 1 (Nxnll) gene has two alternative splice isoforms: a rod-derived cone viability factor that functions in the retina [41]. Inositol hexakisphosphate, the nonvisual arrestin oligomerization and cellular localization are modulated by its binding [42]. In insects, detection of light changes, vibration, colors and semiochemicals, which have evolutionarily old sensory functions, are vital for behaviors including avoiding predation, food location and intraspecific communication. Thus, we speculated that these genes under purifying selection would be important for nervous or visual functions in E. onukii.

We found that $91.8 \%$ of the selected genes (79/86) were under balancing selection with positive Tajima's $D$ values that greatly deviated from zero, indicating that the populations of E. onukii maintained a high level of polymorphism. A much higher genetic diversity ( $\pi=0.00804$ ) was observed in those genomic regions under balancing selection compared to genome-wide diversity $(P<0.0001$, T-test $)$, suggesting a strong capacity for E. onukii to rapidly adapt to diverse habitats [43]. GO enrichment analysis showed that these genes were enriched in several biological processes including cell periphery, plasma membrane part, transmembrane transport, 
ion binding, anion binding, and nucleoside-triphosphatase activity (Figure S10; Table S17). KEGG enrichment analyses pointed to several pathways including those linked to metabolism, circadian rhythms, and immune system functions. enriched (Figure 3B). Previous studies have reported that protein acetylation plays critical roles in cellular processes ranging from gene expression to metabolism [44]. Lysine succinylation is a recently identified post-translational modification (PTM) [45]. It is important for metabolism and detoxification in B. mori [45]. In our study, the apoptosis pathway was consistently enriched (Figure S11). Studies in Lepidoptera insects suggest that apoptosis plays a vital role in resistance to virus infection and some apoptosis-related proteins are known to be succinylated [40, 46, 47]. Here, we identified four key genes, including rve, Pyridoxal_deC and two Glyco_transf_22, which were functionally present in lysine succinylation and glutarylation pathways (Figure 3B).

Lysine succinylation is important for virus-infection resistance and detoxification in insects [44-46, 48, 49]. To examine whether the selected pathways in E. onukii had similar functions, we analyzed the expression patterns of four genes, rve, Pyridoxal_deC and two Glyco_transf_22, collected from 11 different tea cultivars including 4 cultivars (LongJ, DeQ, JianD, JuY) showing resistance to E. onukii and 4 cultivars (ZhuS, LanT, BanZ, EnB) showing susceptibility to E. onukii. Based on RNA-Seq analysis, two genes (Pyridoxal_deC, Glyco_transf_22) showed significantly high expression in both susceptible and resistant tea cultivars, but with different patterns (Figure 3C) $(P<0.05$, T-test). Pyridoxal_deC and Glyco_transf_22 were key genes in metabolic regulation of succinyl- and glutaryl-CoAs (Figure 3B). These results, together with the previously reported roles of succinylation and glutarylation in other insects [44-46, 49], indicated that genes under balancing selection could be involved in metabolic regulation and detoxification of E. onukii, possibly contributing to its success in adapting to a wide range of tea cultivars grown 
in ecologically diverse regions of China.

E. onukii is largely controlled using insecticides in China, leading to development of resistance to chemicals. The ATP-binding cassette (ABC) transporters are conserved across insects and have been implicated in insecticide resistance among pest species [50]. Based on our analyses, the $\mathrm{ABC}$ superfamily showed no expansion, and few orthologs were present in E. onukii compared to other insect species (Table 3). However, we identified four $\mathrm{ABC}$ transporter genes that showed signatures of balancing selection and thus maintaining high genetic variation within populations of E. onukii. Further analysis showed that these four genes belonged to three $\mathrm{ABC}$ subfamilies including $\mathrm{ABCG}, \mathrm{ABCB}$, and $\mathrm{ABCA}$. Balancing selection favors defense proteins with functions in resistance, immunity and adaptations [51, 52]. However, the functions of these four genes have not been elucidated in leafhoppers or aphids. Studies in other animals or insects have shown that these subfamilies are closely related to drug or insecticide resistance $[53,54,55]$. A comparative analysis between susceptible and resistant strains of $A$. aegypti reports that the genes of $\mathrm{ABC}$ transporter G family are highly up-regulated [54]. Similar studies have also been carried out in P. xylostella and L. striatellus, showing that ABCA/B/G subfamilies are significantly over-expressed in the resistant strains $[55,56]$. Based on $\mathrm{ABC}$ family functions in other insects, we hypothesized that E. onukii $\mathrm{ABC}$ genes might contribute to its adaptation to different tea cultivars. This hypothesis may be supported by a study of Cry1Ac resistance in P. xylostella [53]. We therefore investigated the expression patterns of the four genes using E. onukii samples collected from 11 different tea cultivars, as described above. These genes showed moderate expression levels across different developmental stages (Figure S12A) and samples of E. onukii from different tea cultivars (Figure S12B), suggesting that these ABC superfamily members could broadly contribute to adaptation to various tea cultivars and even possibly to chemical resistance. Previous studies also suggest that $\mathrm{ABC}$ transporters are not strictly specific to certain chemicals, implying that $\mathrm{ABC}$ transporters have a broad spectrum of chemical substrates and may act as a basis for cross-resistance of 
multiple chemicals [55].

Genomic regions under balancing selection are functionally important because of their high genetic diversity contributing to adaption to environmental change [43]. Based on our results, we hypothesized that balancing selection might have contributed to the high level of polymorphism in E. onukii populations, facilitating adaptation to diverse environments and tea cultivars.

\section{Evolutionary history is inconsistent between TGL and tea cultivars}

We used high-quality SNPs obtained from the 54 E. onukii samples coming from the different tea-growing regions (Figure 4A) in China (Table S13) to profile their phylogeographical relationships. Phylogenetic analysis and network estimation of the E. onukii samples with E. flavescens and Asymmetrasca sp. as outgroups uncovered three geographically clustered groups (Groups I-III; Figure 4B and Figure S13). Group I contained 4 samples collected from Yunnan province, being the closest to the outgroups. Group II included 28 samples mainly collected from eastern China, including Shandong, Jiangsu, Zhejiang, and Anhui provinces. The remaining 22 samples (i.e., Group III) were mainly from 13 provinces of central and southern China (Figure 4B and Table 4). These results were further supported by genetic structure analysis $(K=3)$ based on the Admixture model (Figure 4C and Figure S14) [57]. Three clustered groups of E. onukii samples (Figure 4B; Table 4) were inconsistent with the current division of the four tea-growing regions (Figure 4A) based on the tea growing history, geographical locations, and tea cultivars [58]. These results suggest a different evolutionary history of E. onukii among these regions. Our analyses of phylogenetic and genetic structure confirmed the genetic differences between group I (samples from Yunnan) and the other groups, as shown in a previous study based on microsatellites [5]. However, this previous study suggested four main genetic groups $(K=4)$ [5]. This may be because the present study collected much more samples around China (54 locations in 22 provinces) than the other one (22 19 locations in 13 provinces) and used a greater number of genetic markers (whole-genome SNPs vs. 
microsatellite markers). We observed that individuals from different groups were interspersed (Figures 4B and C), possibly reflecting gene flow across location, as observed in the previous study [5].

To investigate the genetic divergence, we calculated the average pairwise diversity $(\pi)$ within each of the clustered groups (Table 4). Comparably higher levels of genetic diversity (0.004662 and 0.004744) were observed in Groups II and III than in Group I (0.004062). The high genetic diversity of eastern China and southern-central China may be explained by a geographically wide range and ecologically diverse tea-growing conditions. Further, we found a higher genetic diversity in certain subgroups within the major tea-growing provinces of eastern China and southern-central China. The diversity $(\pi=0.0048)$ in Jiangsu and Zhejiang provinces was higher than the overall diversity of eastern China. and similarly, a higher diversity ( $\pi=0.004939$ ) was found in Anhui and Fujian provinces than in southern-central China, indicating the genetic diversity in these locations. We further analyzed the population differentiation $\left(F_{\mathrm{ST}}\right)$ across different geographically clustered groups and showed a very low $F_{\mathrm{ST}}$ value $(0.005902)$ between Group II and III, indicating their genetically close relationship. In contrast, the $F_{\mathrm{ST}}$ values between Group I and Group II or III were much higher (0.052321 in Group II vs. Group I; 0.043225 in Group III vs. Group I), suggesting that the samples from Yunnan province were genetically distant from the populations in eastern China and southern-central China.

A previous study reports that the structure of male genitalia varies among $E$. onukii locations from eastern China, southern-central China and Yunnan province [3]. Our population genetic analysis also showed genetic differences among these different groups (Figure 4). We speculated that some geographical barriers might have restricted gene flow leading to these differences. Yunnan is surrounded by mountains and rivers as a result of an uplift during the Quaternary and is isolated by the steep Hengduan Mountains. Unlike the clonal propagation of tea cultivars in other regions of China, tea cultivation in Yunnan has depended on seeds from early times [59]. This 
might have prevented the interbreeding of local E. onukii populations with populations from other regions. Similarly, the hilly region between Zhejiang and Fujian might separate E. onukii populations, leading to populations genetically different (Figure 4).

\section{Conclusions}

In this project, we develop a high-quality chromosome-level genome with $92.7 \%$ BUSCO completeness for E. onukii, a species of crucial importance to a widely consumed crop linked to human health. Based on genomic profiling and comparison, we find complex patterns of genomic variation and expansion of gene families associated with evolutionary adaptation to chemosensory reception and xenobiotic detoxification. We identify genomic signatures of balancing selection to reveal the high genetic diversity of resistant genes, underlining their important roles in the adaptive evolution of $E$. onukii. Further, we analyze patterns of variation in genomic sequences from 54 samples and two outgroups, uncovering the population structure and evolutionary history of $E$. onukii across the four different tea-growing regions of China. This work will facilitate functional studies on the adaption of this pest to ecologically diverse habitats, and provide the genomic resources and genetic knowledge for development of sustainable pest management strategy.

\section{Materials and Methods}

Insect colony. E. onukii samples were collected in Fuzhou, Fujian province, southeastern China in July 2017 (on the tea cultivar of Huangdan), and then maintained on tea plants in the laboratory. The insectarium environment was set at 28 $\pm 1^{\circ} \mathrm{C}$ and $60 \pm 5 \% \mathrm{RH}$ with a photoperiod (light: dark $=12: 12$ ).

Genome sequencing and assembly. Since the quality of de novo assembly is sensitive to genomic heterozygosity, genomic DNA of adults was extracted from insects after 12 generations of laboratory inbreeding. Chromosome-level assembly 
414 was performed using Nanopore (Oxford Nanopore Technologies, ONT) with

415 chromatin conformation capture (Hi-C) technologies. The raw ONT reads were 416 self-corrected using CANU version 1.7 [61] with parameter corOutCoverage $=100$,

417 and corrected reads were subject to two widely-used long-read assemblers, wtdbg2

418 [62] and SMARTdenovo (https://github.com/ruanjue/smartdenovo). These two

419 assemblers applied the homopolyer compressed (HPC) k-mer indexing algorithm for

420 sequence alignment and assembly, making the heterozygous regions prone to

421 collapsing. To improve the contiguity of contig assemblies, we used Quickmerge [63]

422 to reconcile wtdbg2 and SMARTdenovo assemblies. Each round of assemblies was

423 inspected through evaluation of N50s based on assembled genome size as well as

424 complete/duplicated BUSCO ratio (Table S3), showing 92.7\% completeness and only

$4252.7 \%$ duplication. It also indicated that the redundant sequences were well-handled in

426 our assembly. The total length of the final contig assembly for E. onukii genome was

$427599 \mathrm{Mb}$ with a contig N50 size of $2.2 \mathrm{Mb}$. Illumina short reads were then used to

428 polish the ONT assembled genome by Pilon [60] with the following parameters:

429 --diploid --threads 6 --changes --tracks --fix bases --verbose --mindepth 4. Hi-C

430 libraries were created from nymphs as previously described [34]. The original

431 cross-linked fragments, also known as chimeric fragments, were then processed into

432 paired-end sequencing libraries and sequenced on the Illumina HiSeq X-10 platform.

433 Paired-end reads were uniquely mapped onto the draft assembly, then 3D-DNA

434 pipeline [64] was recruited to correct any mis-joined contigs by detecting abrupt

435 long-range contact patterns. The Hi-C corrected contigs were further linked into 10

436 pseudo-chromosomes using the ALLHiC pipeline [65]. In total, we generated $65 \mathrm{~Gb}$

437 data $(\sim 109 \times)$ sequences for one cell by for Nanopore ONT and 37 Gb clean data

$438(\sim 61 \times)$ of Illumina X-10 from polishing (Table 1S).

439 Official gene set annotation. Annotation of protein-coding genes was based on $a b$

440 initio gene predictions, transcript evidence and homologous protein evidence, which

441 was all implemented in the GEMOMA computational pipeline [66]. RNA-seq data

442 were generated from every developmental stage (egg, 1st - 5th nymph instar and 
443 adult). Besides, multiple studies have shown that resistance to $E$. onukii varies with 444 different tea cultivars [35, 67]. E. onukii samples were collected from 11 main tea 445 cultivars in China, of which four were susceptible to E. onukii (ZhuS, LanT, BanZ and 446 EnB), four were resistant (LongJ, DeQ, JianD, JuY), and three had unknown 447 resistance status (SuC, $\mathrm{ZiD}$ and ZhongC) [35]. RNA-seq reads were first trimmed 448 using the Trimmomatic program [68] and then mapped to the reference genome using 449 HiSAT2 [69]. During homolog-based prediction, the protein sequences of Drosophila 450 melanogaster, Apis mellifera, Myzus persicae, Acyrthosiphon pisum, Tribolium 451 castaneum, and Bombyx mori were downloaded and aligned to the reference assembly 452 using TBLASTN with e-value $1 \mathrm{e}^{-5}$, and the resulting alignment files were subject to 453 GEMOMA annotation.

454 Orthology and phylogenomics. A total of 19 representative insect species including 455 E. onukii were collected for orthology and phylogenetic analyses (Figure 1). A 456 phylogenetic tree based on a concatenated sequence alignment of the single-copy 457 gene families from E. onukii and other insect species was constructed. We identified 4585,736 single copy genes in these insect genomes using OrthoFinder (version 2.0.0) 459 [70] and performed multiple alignments of the single copy genes from the selected 460 genomes using MAFFT v7.299b [71]. Based on a concatenated sequence alignment, a 461 phylogenetic tree was constructed using RAxML software and the 462 PROTGAMMALGX model [18]. Divergence times of the selected insect species 463 were calculated by PAMLv4.8a momcTREE [72]. The Markov chain Monte Carlo 464 (MCMC) was run for 1,000,000 iterations using a sample frequency of 100 after a 465 burn-in of 2,000 iterations, with the other parameters set as defaults. The following 466 constraints were used for time calibrations: D. melanogaster and A. mellifera 467 divergence time (42.8-83.4 million years ago) [73]. FigTree v1.44 was used to 468 visualize the phylogenetic tree. Gene family expansion and contraction analyses were 469 performed using Café v4.0.1 [74].

470 Gene families. Some gene families with functional importance were selected for 
manual annotation based on the high-quality assembly. Most gene families were annotated using known models from previously annotated genomes including $D$. melanogaster, A. mellifera, M. persicae, A. pisum, C. lectularius and B. mori. Some gene families, which were difficult to identify from automated predictions, were identified based on iterative searching. In brief, BLASTP searches for Hemiptera homologs used queries to search the genomic loci for significant hits $\left(\mathrm{e}<10^{-3}\right.$ ). Further, we recruited hidden Markov models (HMMs) to identify certain domains for these selected gene families based on pfam_scan [75]. Multiple sequence alignments of the selected gene families were obtained with MUSCLE [76] and corrected manually. Phylogenetic analysis was conducted using ML and NJ models, and implemented in MEGA7 for 500 bootstraps [77].

Differential gene expression. RNA-seq data were generated from seven developmental stages (egg, $1^{\text {st }}-5^{\text {th }}$ nymph instars and adult) and the 11 populations of E. onukii collected from different tea cultivars (described in Official gene set annotation section). The RNA-seq reads were trimmed using the Trimmomatic program [68] and mapped back to gene models using bowtie [78]. FPKM was calculated based on the RSEM program [79] implanted in Trinity software [80]. Significantly differentially expressed genes were detected with a cutoff $(P<0.05$ and $\left.\log _{2}^{\text {(change fold) }}>|1|\right)[81]$.

Sample collection, resequencing and SNP calling. Individuals of E. onukii (between 100 and 120) were collected from 54 plantations distributed in four tea-growing regions of China: Southwest region (SWR), South of the Yangtze River region (SYR), North of the Yangtze River region (NYR), and South China region (SER) (Table S13). We also collected two samples, E. flavescens (collected from Canada in vineyards) and Asymmetrasca sp. (collected from Africa), as outgroups (Table S13). For DNA extraction, 50 to 100 individuals were mixed. Genomic DNA extraction, library construction and amplification were performed following standard protocols (Supplemental Notes). All samples were sequenced using the Illumina X-10 platform 
499

500

501

502

503

504

505

506

507

508

509

510

511

512

513

514

515

516

517

518

519

520

521

522

523

524

525

with a paired-end read length of $150 \mathrm{bp}$. The GATK toolkit (version: V 3.5-0-g36282e4) [82] and samtools/bcftools [83] were used to detect variants and SNPs following a series of filtering steps as detailed in a Supplemental Note.

Maximum-likelihood tree inference. The phylogenetic tree was built based on SNPs of single-copy genes. The heterozygous and homozygous SNPs were included in the construction of ML tree. For the heterozygous SNPs, the major alleles that had more reads supported than the minor alleles were retained for further analysis. These SNPs were converted to phylip and aligned in fasta format. The ML (maximum likelihood) tree was constructed using IQ-Tree with a self-estimated best substitution model [84].

Admixture analysis. Ancestral population stratification among the re-sequenced TGL populations was inferred using Admixture software [85]. We estimated the optimal ancestral population structure using ancestral population sizes $K=1-4$ and estimated parameter standard errors based on bootstrapping of 2000.

Diversity statistics. VCFtools v 0.1 .3 [86] was used to calculate population diversity statistics. Genetic differentiation $\left(F_{\mathrm{ST}}\right)$ and the average pairwise diversity index $(\pi)$ were estimated based on a sliding window analysis with $100 \mathrm{~kb}$ window size and 50 $\mathrm{kb}$ step size.

Scanning loci under selective sweeps. To identify candidate genes responsible for reciprocal selection in the TGL populations, we performed the Tajima's $D$ test to identify selective sweeps. Loci with Tajima's $D$ that greatly deviated from 0 proved to be a selection niche in the genome. The Tajima's D statistics were calculated using VCFtools program with a $50 \mathrm{~kb}$ window size and $10 \mathrm{~kb}$ step size. A negative Tajima's $D$ indicates population size expansion and/or purification selection. A significantly positive Tajima's $D$ signifies low levels of low and high frequency polymorphisms, indicating a decrease in population size and/or balancing selection [87]. We used the empirical $5 \%$ windows to indicate the significance. The lowest $5 \%$ windows were considered as purifying selection and the highest $5 \%$ were considered as balancing 
526

527

528

529

530

531

532

533

534

535

536

537

538

539

540

541

542

543

544

545

546

547

548

549

550

551

552

selection.

Based on the annotation of our high-quality genome, candidate genes were identified using our outliers. GO annotation was conducted using Blast2GO [88] and the KEGG pathway analysis was performed using OmicShare tools (www.omicshare.com/tools).

\section{Data availability}

The genome sequences and re-sequencing reads have been deposited in NCBI with accession number of PRJNA731240 and GSA database (https://ngdc.cncb.ac.cn/search/?dbId=gsa\&q=Empoasca) with the accession number of GWHBAZN00000000. Reads for RNA-seq was deposited in GSA database with the accession number of PRJCA005189. The mitochondrial sequence reported in this paper have been deposited in the Genome Warehouse in National Genomics Data Center with the accession number GWHBFSP00000000 that is publicly accessible at https://ngdc.cncb.ac.cn/gwh.

\section{CRediT author statement}

Qian Zhao: Investigation, Methodology, Formal analysis, Visualization, Writing original draft, Writing - review \& editing. Longqing Shi: Resources, Methodology, Writing - original draft. Weiyi He: Methodology, Writing - review \& editing. Jinyu Li: Resources, Formal analysis. Shijun You: Resources, Data curation. Shuai Chen: Formal analysis. Jing Lin: Visualization, Formal analysis. Yibin Wang: Formal analysis. Liwen Zhang: Visualization. Guang Yang: Resources, Writing - review \& editing. Liette Vasseur: Resources, Writing - review \& editing. Minsheng You: Conceptualization, Methodology, Resources, Writing - original draft, Writing - review $\&$ editing, Supervision. All authors read and approved the final manuscript.

\section{Competing interests}

The authors declare that they have no competing interests.

\section{Acknowledgements}


553 This work was supported by The National Key R \& D Program of China 554 (2019YFD1002100), Fujian Agriculture and Forestry University Construction Project 555 for Technological Innovation and Service System of Tea Industry Chain 556 (K1520005A03) and Key International Science and Technology cooperation Project 557 of China (2016YFE0102100). We thank Mr. Haifang He, and Fasheng Huang for their 558 kind assistance in collection of the insect samples.

\section{ORCID}

560 0000-0003-4256-5686 (Qian Zhao)

561 0000-0003-2036-7558 (Longqing Shi)

562 0000-0001-8659-3123 (Weiyi He)

563 0000-0003-4560-3190 (Jinyu Li)

564 0000-0001-7340-1524 (Shijun You)

565 0000-0002-6861-2682 (Shuai Chen)

566 0000-0002-5913-1801 (Jing Lin)

567 0000-0002-0781-3966 (Yibin Wang)

568 0000-0001-9220-3849 (Liwen Zhang)

569 0000-0002-3250-5228 (Guang Yang)

570 0000-0001-7289-2675 (Liette Vasseur)

571 0000-0001-9042-6432 (Minsheng You) 
572

573

574

575

576

577

578

579

580

581

582

583

584

585

586

587

588

589

590

591

592

593

594

595

596

597

598

599

600

\section{References}

[1] Fu JY, Han BY and Xiao Q. Mitochondrial COI and 16sRNA Evidence for a Single Species Hypothesis of E. vitis, J. formosana and E. onukii in East Asia. PLoS One 2014; 9(12): p. e115259.

[2] Chen LL, Yuan P, Pozsgai G, Chen P, Zhu H and You MS. The impact of cover crops on the predatory mite Anystis baccarum (Acari, Anystidae) and the leafhopper pest Empoasca onukii (Hemiptera, Cicadellidae) in a tea plantation. Pest Manag Sci 2019; 75(12): p. 3371-3380.

[3] Qin D, Zhang L, Xiao Q, Dietrich C and Matsumura M. Clarification of the Identity of the Tea Green Leafhopper Based on Morphological Comparison between Chinese and Japanese Specimens. PLoS One 2015; 10(9): p. e0139202.

[4] Lv WM, Chen X and Luo QR. Research on occurrence and control of Empoasca flavescens. Journal of Tea Science 1964: p. 45-55.

[5] Zhang L, Wang F, Qiao L, Dietrich CH, Matsumura M and Qin D. Population structure and genetic differentiation of tea green leafhopper, Empoasca (Matsumurasca) onukii, in China based on microsatellite markers. Sci Rep 2019; 9(1): p. 1202.

[6] Xiao Z, Huang X, Zang Z and Yang H. Spatio-temporal variation and the driving forces of tea production in China over the last 30 years. J. Geogr. Sci 2018; 28: p. 275-290.

[7] Panfilio KA, Vargas Jentzsch IM, Benoit JB, Erezyilmaz D, Suzuki Y, Colella S, et al. Molecular evolutionary trends and feeding ecology diversification in the Hemiptera, anchored by the milkweed bug genome. Genome Biol 2019; 20(1): p. 64 .

[8] Jin S, Sun X, Chen Z and Xiao B. Resistance of the tea green leafhopper to different tea plant varieties. Sci. Agric. Sin 2012; 45(2): p. 255-265.

[9] Rosenfeld JA, Reeves D, Brugler MR, Narechania A, Simon S, Durrett R, et al. Genome assembly and geospatial phylogenomics of the bed bug Cimex lectularius. Nat Commun 2016; 7: p. 10164.

[10] J. A. Wenger, B. J. Cassone, F. Legeai, J. S. Johnston, R. Bansal, A. D. Yates, et al. Whole genome sequence of the soybean aphid, Aphis glycines. Insect Biochem Mol Biol 2017.

[11] Ou S, Chen J and Jiang N. Assessing genome assembly quality using the LTR Assembly Index (LAI). Nucleic Acids Res 2018; 46(21): p. e126. 
601 [12] Nicholson SJ, Nickerson ML, Dean M, Song Y, Hoyt PR, Rhee H, et al. The genome of

602 Diuraphis noxia, a global aphid pest of small grains. BMC Genomics 2015; 16: p. 429.

603 [13] Li Y, Park H, Smith TE and Moran NA. Gene Family Evolution in the Pea Aphid Based on

604 Chromosome-Level Genome Assembly. Mol Biol Evol 2019; 36(10): p. 2143-2156.

605 [14] Ye YX, Zhang HH, Li DT, Zhuo JC, Shen Y, Hu QL, et al. Chromosome-level assembly of

606 the brown planthopper genome with a characterized Y chromosome. Mol Ecol Resour 2021; 21(4):

607 p. $1287-1298$.

608 [15] Xue J, Zhou X, Zhang CX, Yu LL, Fan HW, Wang Z, et al. Genomes of the rice pest brown 609 planthopper and its endosymbionts reveal complex complementary contributions for host 610 adaptation. Genome Biol 2014; 15(12): p. 521.

611 [16] Kapusta A, Suh A and Feschotte C. Dynamics of genome size evolution in birds and 612 mammals. Proc Natl Acad Sci U S A 2017; 114(8): p. E1460-E1469.

613 [17] Wolfson RL, Chantranupong L, Wyant GA, Gu X, Orozco JM, Shen K, et al. KICSTOR 614 recruits GATOR1 to the lysosome and is necessary for nutrients to regulate mTORC1. Nature $615 \quad 2017 ; 543(7645):$ p. 438-442.

616 [18] Stamatakis A. RAxML version 8: a tool for phylogenetic analysis and post-analysis of large 617 phylogenies. Bioinformatics 2014; 30(9): p. 1312-3.

618 [19] F. Cui, M. X. Li, H. J. Chang, Y. Mao, H. Y. Zhang, L. X. Lu, et al. 619 Carboxylesterase-mediated insecticide resistance: Quantitative increase induces broader metabolic 620 resistance than qualitative change. Pestic Biochem Physiol 2015; 121: p. 88-96.

621 [20] S. Cheng, J. Ashley, J. D. Kurleto, M. Lobb-Rabe, Y. J. Park, R. A. Carrillo, et al. Molecular 622 basis of synaptic specificity by immunoglobulin superfamily receptors in Drosophila. Elife 2019; 6238.

624 [21] C. Xu, E. Theisen, R. Maloney, J. Peng, I. Santiago, C. Yapp, et al. Control of Synaptic 625 Specificity by Establishing a Relative Preference for Synaptic Partners. Neuron 2020; 106(2): p. 626355.

627 [22] Dahanukar A, Hallem EA and Carlson JR. Insect chemoreception. Curr Opin Neurobiol 2005; 628 15(4): p. 423-30.

629 [23] Bargmann CI. Comparative chemosensation from receptors to ecology. Nature 2006; 630 444(7117): p. 295-301. 
631 [24] Wang Q, Zhou JJ, Liu JT, Huang GZ, Xu WY, Zhang Q, et al. Integrative transcriptomic and

632 genomic analysis of odorant binding proteins and chemosensory proteins in aphids. Insect Mol

633 Biol 2019; 28(1): p. 1-22.

634 [25] Youn YN. Electroantennogram responses of Nilaparvata lugens (Homoptera: Delphacidae) to 635 plant volatile compounds. J Econ Entomol 2002; 95(2): p. 269-77.

636 [26] He P, Zhang J, Liu NY, Zhang YN, Yang K and Dong SL. Distinct expression profiles and 637 different functions of odorant binding proteins in Nilaparvata lugens Stal. PLoS One 2011; 6(12): 638 p. e28921.

639 [27] Xue J, Zhou X, Zhang XC, Yu LL, Fan HW, Wang Z, et al. Genomes of the rice pest brown 640 planthopper and its endosymbionts reveal complex complementary contributions for host 641 adaptation. Genome Biol 2014; 15: p. 521.

642 [28] Robertson HM, Robertson ECN, Walden KKO, Enders LS and Miller NJ. The 643 chemoreceptors and odorant binding proteins of the soybean and pea aphids. Insect Biochem Mol $644 \quad$ Biol 2019; 105: p. 69-78.

645 [29] Pearce SL, Clarke DF, East PD, Elfekih S, Gordon KHJ, Jermiin LS, et al. Genomic 646 innovations, transcriptional plasticity and gene loss underlying the evolution and divergence of 647 two highly polyphagous and invasive Helicoverpa pest species. BMC Biol 2017; 15(1): p. 63.

648 [30] McBride CS. Rapid evolution of smell and taste receptor genes during host specialization in 649 Drosophila sechellia. Proc Natl Acad Sci U S A 2007; 104(12): p. 4996-5001.

650 [31] Benton R, Vannice KS, Gomez-Diaz C and Vosshall LB. Variant ionotropic glutamate 651 receptors as chemosensory receptors in Drosophila. Cell 2009; 136(1): p. 149-62.

652 [32] Chen C, Buhl E, Xu M, Croset V, Rees JS, Lilley KS, et al. Drosophila Ionotropic Receptor 65325 a mediates circadian clock resetting by temperature. Nature 2015; 527(7579): p. 516-20.

654 [33] Wei Q, Yu HY, Niu CD, Yao R, Wu SF, Chen Z, et al. Comparison of Insecticide 655 Susceptibilities of Empoasca vitis (Hemiptera: Cicadellidae) from Three Main Tea-Growing 656 Regions in China. J Econ Entomol 2015; 108(3): p. 1251-9.

657 [34] Wan F, Yin C, Tang R, Chen M, Wu Q, Huang C, et al. A chromosome-level genome 658 assembly of Cydia pomonella provides insights into chemical ecology and insecticide resistance. $659 \quad$ Nat Commun 2019; 10(1): p. 4237.

660 [35] Jin S, Sun XL, Chen Z and Xiao B. Resistance of different tea cultivars to Emposca vitis 
661

662

663

664

665

666

667

668

669

670

671

672

673

674

675

676

677

678

679

680

681

682

683

684

685

686

687

688

689

690

gOTHE. Sci. Agric. Sin 2012; 45(2): p. 255-265.

[36] Feyereisen R. Evolution of insect P450. Biochem Soc Trans 2006; 34(Pt 6): p. 1252-5.

[37] Simpson AE. The cytochrome P450 4 (CYP4) family. Gen Pharmacol 1997; 28(3): p. 351-9.

[38] Cvijovic I, Good BH and Desai MM. The Effect of Strong Purifying Selection on Genetic Diversity. Genetics 2018; 209(4): p. 1235-1278.

[39] Braccioli L, Nijboer CH and Coffer PJ. Forkhead box protein P1, a key player in neuronal development? Neural Regen Res 2018; 13(5): p. 801-802.

[40] Chen Y, Ip FC, Shi L, Zhang Z, Tang H, Ng YP, et al. Coronin 6 regulates acetylcholine receptor clustering through modulating receptor anchorage to actin cytoskeleton. J Neurosci 2014; 34(7): p. 2413-21.

[41] Byrne LC, Dalkara D, Luna G, Fisher SK, Clerin E, Sahel JA, et al. Viral-mediated RdCVF and RdCVFL expression protects cone and rod photoreceptors in retinal degeneration. J Clin Invest 2015; 125(1): p. 105-16.

[42] Milano SK, Kim YM, Stefano FP, Benovic JL and Brenner C. Nonvisual arrestin oligomerization and cellular localization are regulated by inositol hexakisphosphate binding. J Biol Chem 2006; 281(14): p. 9812-23.

[43] Wu J, Wang Y, Xu J, Korban SS, Fei Z, Tao S, et al. Diversification and independent domestication of Asian and European pears. Genome Biol 2018; 19(1): p. 77.

[44] Hirschey MD and Zhao Y. Metabolic Regulation by Lysine Malonylation, Succinylation, and Glutarylation. Mol Cell Proteomics 2015; 14(9): p. 2308-15.

[45] Chen J, Li F, Liu Y, Shen W, Du X, He L, et al. Systematic identification of mitochondrial lysine succinylome in silkworm (Bombyx mori) midgut during the larval gluttonous stage. J Proteomics 2018; 174: p. 61-70.

[46] Cheng Y, Wang XY, Hu H, Killiny N and Xu JP. A hypothetical model of crossing Bombyx mori nucleopolyhedrovirus through its host midgut physical barrier. PLoS One 2014; 9(12): p. e115032.

[47] Wang XY, Yu HZ, Xu JP, Zhang SZ, Yu D, Liu MH, et al. Comparative Subcellular Proteomics Analysis of Susceptible and Near-isogenic Resistant Bombyx mori (Lepidoptera) Larval Midgut Response to BmNPV infection. Sci Rep 2017; 7: p. 45690.

[48] Gu Z, Zhou Y, Xie Y, Li F, Ma L, Sun S, et al. The adverse effects of phoxim exposure in the 
691

692

693

694

695

696

697

698

699

700

701

702

703

704

705

706

707

708

709

710

711

712

713

714

715

716

717

718

719

720

midgut of silkworm, Bombyx mori. Chemosphere 2014; 96: p. 33-8.

[49] Sagisaka A, Fujita K, Nakamura Y, Ishibashi J, Noda H, Imanishi S, et al. Genome-wide analysis of host gene expression in the silkworm cells infected with Bombyx mori nucleopolyhedrovirus. Virus Res 2010; 147(2): p. 166-75.

[50] Rosner J and Merzendorfer $\mathrm{H}$. Transcriptional plasticity of different ABC transporter genes from Tribolium castaneum contributes to diflubenzuron resistance. Insect Biochem Mol Biol 2020; 116: p. 103282.

[51] Koenig D, Hagmann J, Li R, Bemm F, Slotte T, Neuffer B, et al. Long-term balancing selection drives evolution of immunity genes in Capsella. Elife 2019; 8 .

[52] Van der Hoorn RA, De Wit PJ and Joosten MH. Balancing selection favors guarding resistance proteins. Trends Plant Sci 2002; 7(2): p. 67-71.

[53] Ocelotl J, Sanchez J, Gomez I, Tabashnik BE, Bravo A and Soberon M. ABCC2 is associated with Bacillus thuringiensis Cry1Ac toxin oligomerization and membrane insertion in diamondback moth. Sci Rep 2017; 7(1): p. 2386.

[54] Lien NTK, Ngoc NTH, Lan NN, Hien NT, Tung NV, Ngan NTT, et al. Transcriptome Sequencing and Analysis of Changes Associated with Insecticide Resistance in the Dengue Mosquito (Aedes aegypti) in Vietnam. Am J Trop Med Hyg 2019; 100(5): p. 1240-1248.

[55] Sun H, Pu J, Chen F, Wang J and Han Z. Multiple ATP-binding cassette transporters are involved in insecticide resistance in the small brown planthopper, Laodelphax striatellus. Insect Mol Biol 2017; 26(3): p. 343-355.

[56] You M, Yue Z, He W, Yang X, Yang G, Xie M, et al. A heterozygous moth genome provides insights into herbivory and detoxification. Nat Genet 2013; 45(2): p. 220-5.

[57] Pritchard JK, Stephens M and Donnelly P. Inference of population structure using multilocus genotype data. Genetics 2000; 155(2): p. 945-59.

[58] Zhang WJ, Rong J, Wei CL, Gao LP and Chen JK. Domestication origin and spread of cultivated tea plants. Biodiversity Science 2018; 26(4): p. 357-372.

[59] Preparation committee, Records of tea varieties in China. 2001, Shanghai: Shanghai Scientific \& Technical Publishers.

[60] Walker BJ, Abeel T, Shea T, Priest M, Abouelliel A, Sakthikumar S, et al. Pilon: an integrated tool for comprehensive microbial variant detection and genome assembly improvement. PLoS 
One 2014; 9(11): p. e112963.

[61] Koren S, Walenz BP, Berlin K, Miller JR, Bergman NH and Phillippy AM. Canu: scalable and accurate long-read assembly via adaptive k-mer weighting and repeat separation. Genome Res 2017; 27(5): p. 722-736.

[62] Ruan J and Li H. Fast and accurate long-read assembly with wtdbg2. Nat Methods 2020; 17(2): p. $155-158$.

[63] Chakraborty M, Baldwin-Brown JG, Long AD and Emerson JJ. Contiguous and accurate de novo assembly of metazoan genomes with modest long read coverage. Nucleic Acids Res 2016; 44(19): p. e147.

[64] Dudchenko O, Batra SS, Omer AD, Nyquist SK, Hoeger M, Durand NC, et al. De novo assembly of the Aedes aegypti genome using Hi-C yields chromosome-length scaffolds. Science 2017; 356(6333): p. 92-95.

[65] Zhang X, Zhang S, Zhao Q, Ming R and Tang H. Assembly of allele-aware, chromosomal-scale autopolyploid genomes based on Hi-C data. Nat Plants 2019; 5(8): p. 833-845. [66] Keilwagen J, Hartung F, Paulini M, Twardziok SO and Grau J. Combining RNA-seq data and homology-based gene prediction for plants, animals and fungi. BMC Bioinformatics 2018; 19(1): p. 189.

[67] Miao J, Han BY and Zhang QH. Probing behavior of Empoasca vitis (Homoptera: Cicadellidae) on resistant and susceptible cultivars of tea plants. J Insect Sci 2014; 14.

[68] Bolger AM, Lohse M and Usadel B. Trimmomatic: a flexible trimmer for Illumina sequence data. Bioinformatics 2014; 30(15): p. 2114-20.

[69] Kim D, Paggi JM, Park C, Bennett C and Salzberg SL. Graph-based genome alignment and genotyping with HISAT2 and HISAT-genotype. Nat Biotechnol 2019; 37(8): p. 907-915.

[70] Emms DM and Kelly S. OrthoFinder: phylogenetic orthology inference for comparative genomics. Genome Biol 2019; 20(1): p. 238.

[71] Nakamura T, Yamada KD, Tomii K and Katoh K. Parallelization of MAFFT for large-scale multiple sequence alignments. Bioinformatics 2018; 34(14): p. 2490-2492.

[72] Yang Z. PAML: a program package for phylogenetic analysis by maximum likelihood. Comput Appl Biosci 1997; 13(5): p. 555-6.

[73] Xiao JH, Yue Z, Jia LY, Yang XH, Niu LH, Wang Z, et al. Obligate mutualism within a host 
drives the extreme specialization of a fig wasp genome. Genome Biol 2013; 14(12): p. R141.

[74] De-Bie T, Cristianini N, Demuth JP and Hahn MW. CAFE: a computational tool for the study of gene family evolution. Bioinformatics 2006; 22(10): p. 1269-71.

[75] Eddy SR. Profile hidden Markov models. Bioinformatics 1998; 14(9): p. 755-63.

[76] Edgar RC. MUSCLE: multiple sequence alignment with high accuracy and high throughput. Nucleic Acids Res 2004; 32(5): p. 1792-7.

[77] Hall BG. Building phylogenetic trees from molecular data with MEGA. Mol Biol Evol 2013; 30(5): p. 1229-35.

[78] Langmead B, Trapnell C, Pop M and Salzberg SL. Ultrafast and memory-efficient alignment of short DNA sequences to the human genome. Genome Biol 2009; 10(3): p. R25.

[79] Li B and Dewey CN. RSEM: accurate transcript quantification from RNA-Seq data with or without a reference genome. BMC Bioinformatics 2011; 12: p. 323.

[80] Haas BJ, Papanicolaou A, Yassour M, Grabherr M, Blood PD, Bowden J, et al. De novo transcript sequence reconstruction from RNA-seq using the Trinity platform for reference generation and analysis. Nat Protoc 2013; 8(8): p. 1494-512.

[81] Montgomery SH and Mank JE. Inferring regulatory change from gene expression: the confounding effects of tissue scaling. Mol Ecol 2016; 25(20): p. 5114-5128.

[82] McKenna A, Hanna M, Banks E, Sivachenko A, Cibulskis K, Kernytsky A, et al. The Genome Analysis Toolkit: a MapReduce framework for analyzing next-generation DNA sequencing data. Genome Res 2010; 20(9): p. 1297-303.

[83] Li H, Handsaker B, Wysoker A, Fennell T, Ruan J, Homer N, et al. The Sequence Alignment/Map format and SAMtools. Bioinformatics 2009; 25(16): p. 2078-9.

[84] Nguyen LT, Schmidt HA, von Haeseler A and Minh BQ. IQ-TREE: a fast and effective stochastic algorithm for estimating maximum-likelihood phylogenies. Mol Biol Evol 2015; 32(1): p. $268-74$.

[85] Patterson N, Moorjani P, Luo Y, Mallick S, Rohland N, Zhan Y, et al. Ancient admixture in human history. Genetics 2012; 192(3): p. 1065-93.

[86] Danecek P, Auton A, Abecasis G, Albers CA, Banks E, DePristo MA, et al. The variant call format and VCFtools. Bioinformatics 2011; 27(15): p. 2156-8.

[87] Kreitman M. Methods to detect selection in populations with applications to the human. Annu. 
bioRxiv preprint doi: https://doi.org/10.1101/2021.11.23.469662; this version posted November 23, 2021. The copyright holder for this preprint (which was not certified by peer review) is the author/funder, who has granted bioRxiv a license to display the preprint in perpetuity. It is made available under aCC-BY-NC-ND 4.0 International license.

781 Rev. Genomics Hum. Genet. 2000; 01: p. 539-59.

782 [88] Conesa A, Gotz S, Garcia-Gomez JM, Terol J, Talon M and Robles M. Blast2GO: a

783 universal tool for annotation, visualization and analysis in functional genomics research.

784 Bioinformatics 2005; 21(18): p. 3674-6.

785 


\section{Figure legends}

787

788

789

790

791

792

793

794

795

796

797

798

799

800

801

802

803

804

805

806

807

808

809

810

811

812

813

Figure 1. Genomic characterization of $E$. onukii and comparison with other insect genomes.

(A) Genomic characterization of the sequenced E. onukii. The circles (from outermost to innermost) represent monoploid genome in $\mathrm{Mb}$, segmental duplication, gene density, LTR Copia/Gypsy, DNA transposable elements and expression profiles. (B) Numerical comparison of homologous genes between gene sets from E. onukii and each of the five Hemiptera species. Dataset overlaps were determined using a BLASTP search $\left(e\right.$ value $\left.<10^{-5}\right)$. (C) Phylogenetic relationships among 15 insect species based on genomic comparisons. Single copy orthologs: only one copy in different genomes, multicopy orthologs: more than one copy in different genomes, unique paralogs: species-specific genes, other orthologs: unclassified orthologs, unclustered genes: genes that cannot be clustered into known gene families. Details about the identification are previously described [73].

Figure 2. Expansion of gene families related to chemoreception and detoxification in $E$. onukii compared with other insect species.

(A) Numerical comparison of the chemosensory proteins (CSPs) among aphids, plant bugs and hoppers. Aphid species include Acyrthosiphon pisum (Apis), Myzus persicae (Mper), Aphis gossypii (Agos), Aphis glycines (Agly) and Sitobion avenae (Save); Plant bugs include Adelphocoris lineolatus (Alin), Adelphocoris suturalis (Asut), Apolygus lucorum (Aluc); Hoppers includes Empoasca onukii (Eonu), Laodelphax striatellus (Lstr), Nilaparvata lugens (Nlug) and Sogatella furcifera (Sfur). (B) Phylogenetic relationships of CSPs in hoppers (N. lugens, S. furcifera, and $L$. striatellus), aphids (A. pisum, M. persicae, A. gossypii, A. glycines, and S. avenae), and plant bugs ( $N$. lugens, L. striatellus and S. furcifera). Yellow branches represent CSP family genes in E. onukii. (C) Genomic expansion and unbalanced chromosomal distribution of CSPs in the E. onukii genome. (D) Phylogenetic relationships and expression profiling of detoxification-related proteins (CYP3 and CYP4) in plant 
814 hoppers, aphids and plant bugs. Expression profiling based on RNA-seq data were

815 generated from all developmental stages (egg, 1st -5 th nymph instar and adult) and 8

816 E. onukii populations collected from different tea cultivars (four cultivars being

817 resistant including LongJ, DeQ, JianD, JuY and four cultivars susceptible to E. onukii

818 including ZhuS, LanT, BanZ and EnB).

\section{Figure 3. Genomic signatures of balancing selection.}

820 (A) Putative selection sweeps in populations of E. onukii. Tajima's $D$ value was 821 calculated for each of the E. onukii populations. Mean values of Tajima's $D$ are shown 822 in sliding windows of $50 \mathrm{~kb}$ with a step size of $10 \mathrm{~kb}$. Regions with Tajima's $D$ values

823 deviated significantly from 0 are marked with dotted lines in panel 1. Specifically, 824 values of Tajima's $D$ significantly deviated from 0 are plotted in red (>0) and green 825 (<0) respectively in panel 2 and panel 3. (B) Succinyl- and glutaryl-CoA pathways 826 showing the regulatory role of lysine modifications in metabolism. (C) Expression 827 patterns of the E. onukii genes under balancing selection, in 11 different tea cultivars. 828 Pyridoxal_deC was detected to be significantly highly expressed in susceptible tea 829 cultivars $(P<0.01$, T-test), while Glyco_transf_22 was significantly highly expressed 830 in resistant tea cultivars $(P<0.05$, T-test $)$.

Figure 4. Phylogenetic relationship, population structure and expansion of $E$. 832 onukii.

833 (A) Geographical locations (sites) of 54 samples collected from four tea-growing 834 regions around China: Southwest region (SWR), South of the Yangtze River region 835 (SYR), North of the Yangtze River region (NYR), and South China region (SER). 836 Dots with different colors represent different clustered groups. (B) Phylogenetic tree 837 of the 54 E. onukii samples based on RAxML and SplitsTress. Branch lengths are not 838 scaled. Different colors of inner circle represent 4 different tea-growing regions 839 shown in (A). Colors of outer lane represent different E. onukii groups based on 840 phylogenetic analysis. (C) Genetic structure and individual ancestry with colors in 841 each column representing ancestry proportion over range of population sizes $(K=2-4$, 
842 with an optimal $K=3$ ).

843 Table legends

844 Table 1. Sequencing, chromosome-scale assembly and annotation of the E. onukii

845 genome

846 Table 2. Chromosome-based statistics of the $E$. onukii genome

847 Table 3. Numerical comparison of genes related to chemoreception and 848 detoxification among different insect species

849 Table 4. Number of populations, nuclear SNPs, and genetic diversity $(\pi)$ in each 850 of the three clustered groups

851 Supplementary material

852 Title for supplementary file: Supplemental_Notes.docx

853 Figure legends:

854 Figure S1. Damages of E.onukii in modern tea plantation in China. (A) Life cycle 855 of E. onukii. (B) Damages caused by E.onukii. (C) Developmental stages of E. onukii.

856 Figure S2. Genome assembly of $E$. onukii. (A) Chromatin interactions with 150

857 kb resolution in $E$. onukii. (B) Mitochondrial genome of $E$. onukii. Inner circle

858 represents the GC content while outer circle represents genes located on

859 mitochondrion.

860 Figure S3. (A) Assessment of the assembly using LTR Assembly Index (LAI). (B)

861 Correlation analysis between repeat content and the genome size.

862 Figure S4. Gene family expansions and contractions in the $E$. onukii compared 863 with other insects. Numbers for expanded (green) and contracted (red) gene families 864 are shown on branches.

865 Figure S5. Expression profile of genes involved in chemoreception and 866 phylogenetic analysis of odorant-binding proteins (OBPs). (A) Expression of 5 867 chemoreception gene families. (B) Neighbor-joining method involving 84 protein 868 sequences was used to construct the tree. Different colors represent different species: 
869

870

871

872

873

874

875

red represents Ap. Glycines (Agly); green represents A. pisum (Apis); $\quad$ purple represents E. onukii (Em) while black represents D. melanogaster.

Figure S6. Phylogenetic analysis of olfactory receptors (ORs). Neighbor-joining method involving 216 protein sequences was used to construct the tree. Different colors represent different species: red represents Ap. Glycines (Agly); green represents A. pisum (Apis); yellow represents E. onukii (Em) while blue represents D. melanogaster.

Figure S7. Phylogenetic analysis of gustatory receptors (GRs). Neighbor-joining method involving 219 protein sequences was used to construct the tree. Colors corresponded to Supplemental Figure 6.

Figure S8. Phylogenetic analysis of ionotropic receptors (IRs). Neighbor-joining method involving 125 protein sequences was used to construct the tree. Colors corresponded to Supplemental Figure 6.

Figure S9. Phylogenetic analysis of $\mathbf{P 4 5 0}$ gene family. Neighbor-joining method involving 259 protein sequences was used to construct the tree. Colors represented different species listed in the Figure.

Figure S10. GO enrichment of the genes under balancing selection in E. onukii. Only top 20 were listed in the figures.

Figure S11. Apoptosis pathway was enriched for the genes under balancing selection in $\boldsymbol{E}$. onukii. Genes under selection were marked in red.

Figure S12. Expression patterns of the ABC genes under balancing selection. (A) Expression patterns of different developmental stages; (B) Expression patterns when living on resistant and susceptible tea cultivars.

Figure S13. Phylogenetic tree and network estimation by RAxML and SplitsTress. Three populations according to the geographic regions that most of the individuals located: group I was Yunan (YN), Eastern China (group II); and central and southern of China (group III) were listed in Red, Green, Blue respectively (right figure) with presence of the outgroups (left figure). Different 4 tea regions of China were listed in different colors represented in Figure 3.

Figure S14. Admixture analysis of 55 TGLs accessions $(k=2-4)$. IDs were 
899 represented in Supplemental Table 12.

900 Table legends

901 Table S1. Statistics of genomic sequencing data of Empoasca onukii

902 Table S2. Statistics of Hi-C mapping

903 Table S3. Statistics of contig level assembly of $E$. onukii

904 Table S4. BUSCO analysis of genome assembly of $E$. onukii

905 Table S5. BUSCO analysis of annotation completeness

906 Table S6. The statistics of different Hemiptera species assembly

907 Table S7. Assessment of genome consistency based on NGS (Illumina) reads

908 Table S8. Statistics of TEs in E. onukii genome

909 Table S9. GO over-representation of gene families expanded on E.onukii branch

910 Table S10. Gene family contraction analysis on E.onukii branch

911 Table S11. Chemosensory related gene families in $E$. onukii

912 Table S12. List of gene families involved in detoxification

913 Table S13. Geographic distributions of the collected samples around China

914 Table S14. Genomic regions under selection

915 Table S15. Genes under selection

916 Table S16. Functional analysis of genes under purifying selection

917 Table S17. GO terms for the genes under balancing selection 


\section{$1 \quad$ Figures}
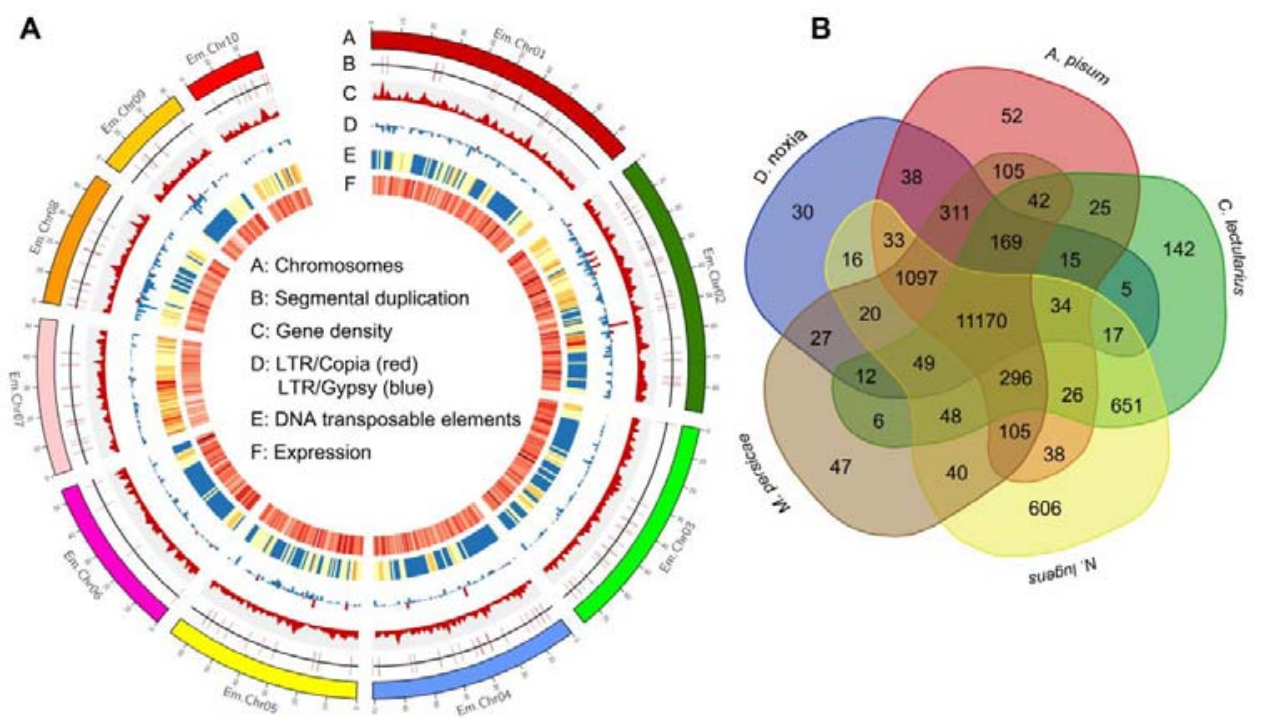

C

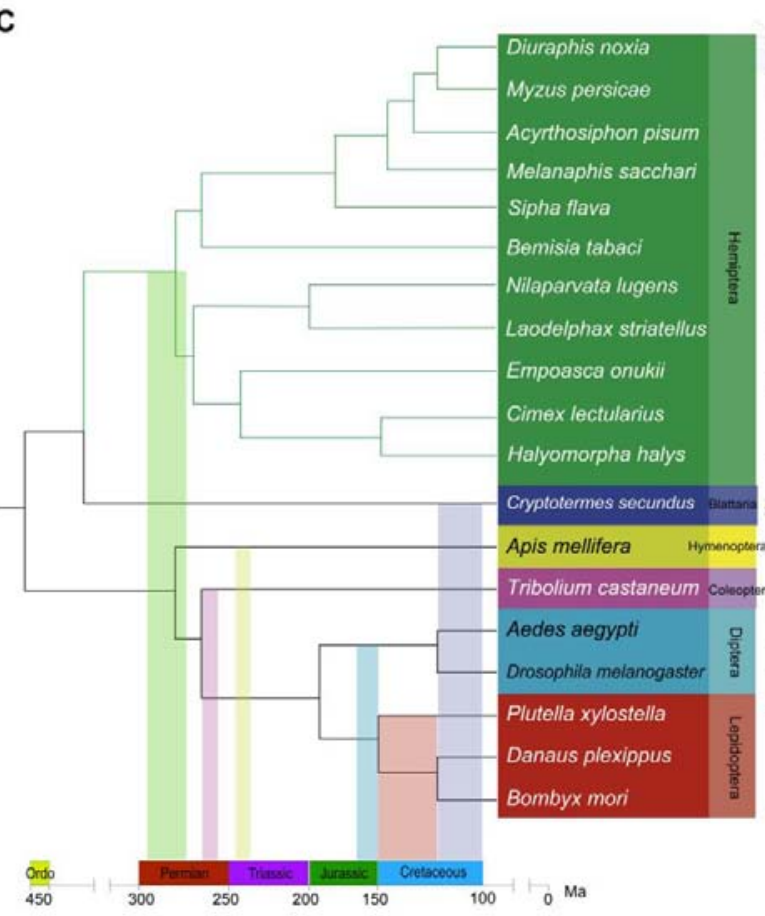

Repetitive content

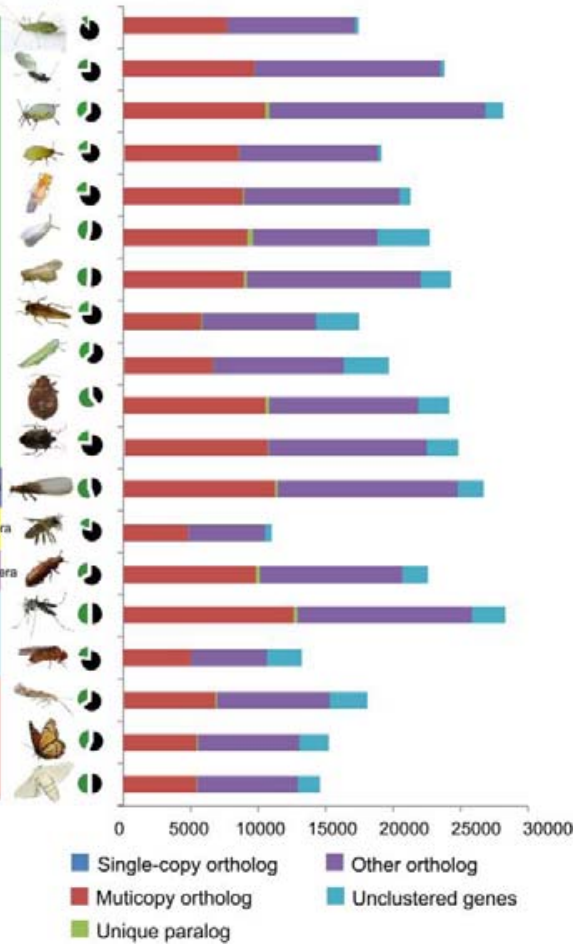

2

Figure 1. Genomic characterization of $E$. onukii and comparison with other insect genomes.

5 (A) Genomic characterization of the sequenced E. onukii. The circles (from outermost 
6 to innermost) represent monoploid genome in $\mathrm{Mb}$, segmental duplication, gene

7 density, LTR Copia/Gypsy, DNA transposable elements and expression profiles. (B)

8 Numerical comparison of homologous genes between gene sets from E. onukii and

9 each of the five Hemiptera species. Dataset overlaps were determined using a

10 BLASTP search $\left(e\right.$ value $\left.<10^{-5}\right)$. (C) Phylogenetic relationships among 15 insect

11 species based on genomic comparisons. Numbers for expanded (green) and contracted

12 (red) gene families are shown on branches. Single copy orthologs: only one copy in

13 different genomes, multicopy orthologs: more than one copy in different genomes,

14 unique paralogs: species-specific genes, other orthologs: unclassified orthologs,

15 unclustered genes: genes that cannot be clustered into known gene families. Details

16 about the identification are previously described [73]. 
A

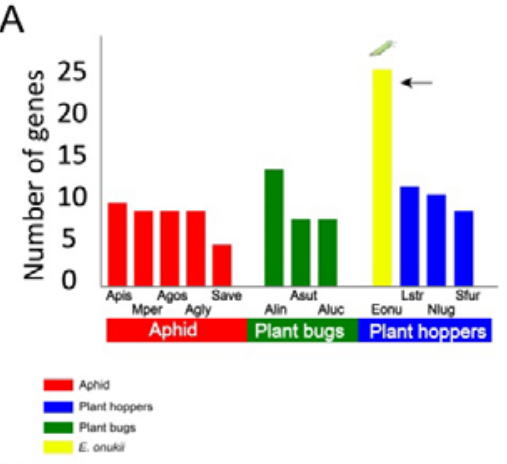

B

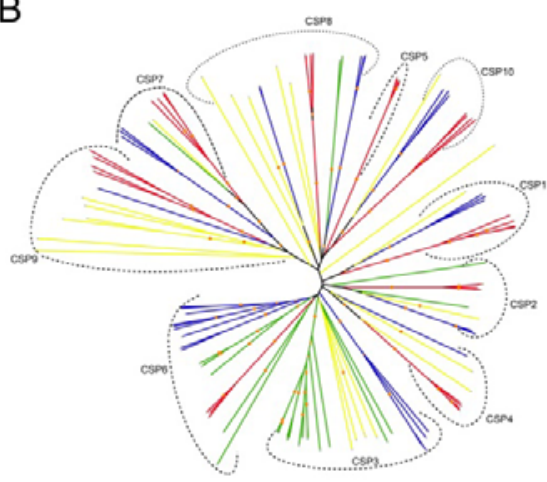

C

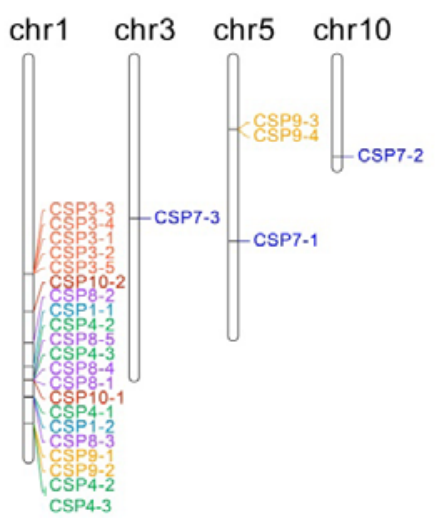

D
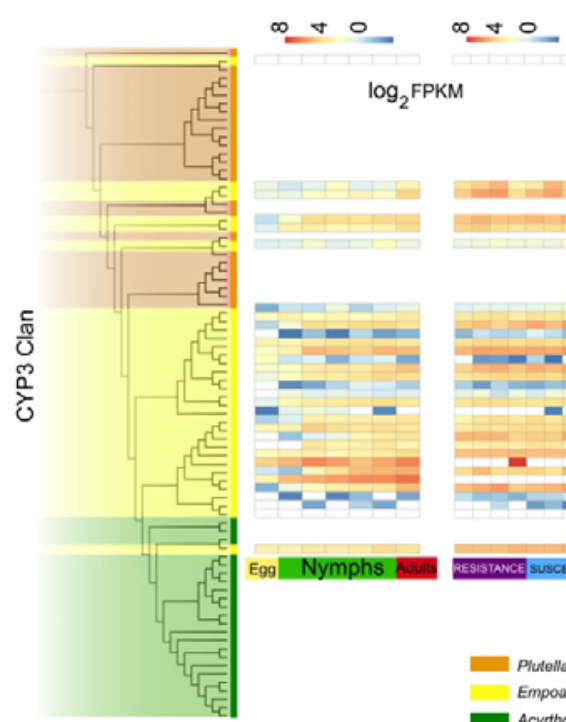

$\log _{2}$ FPKM
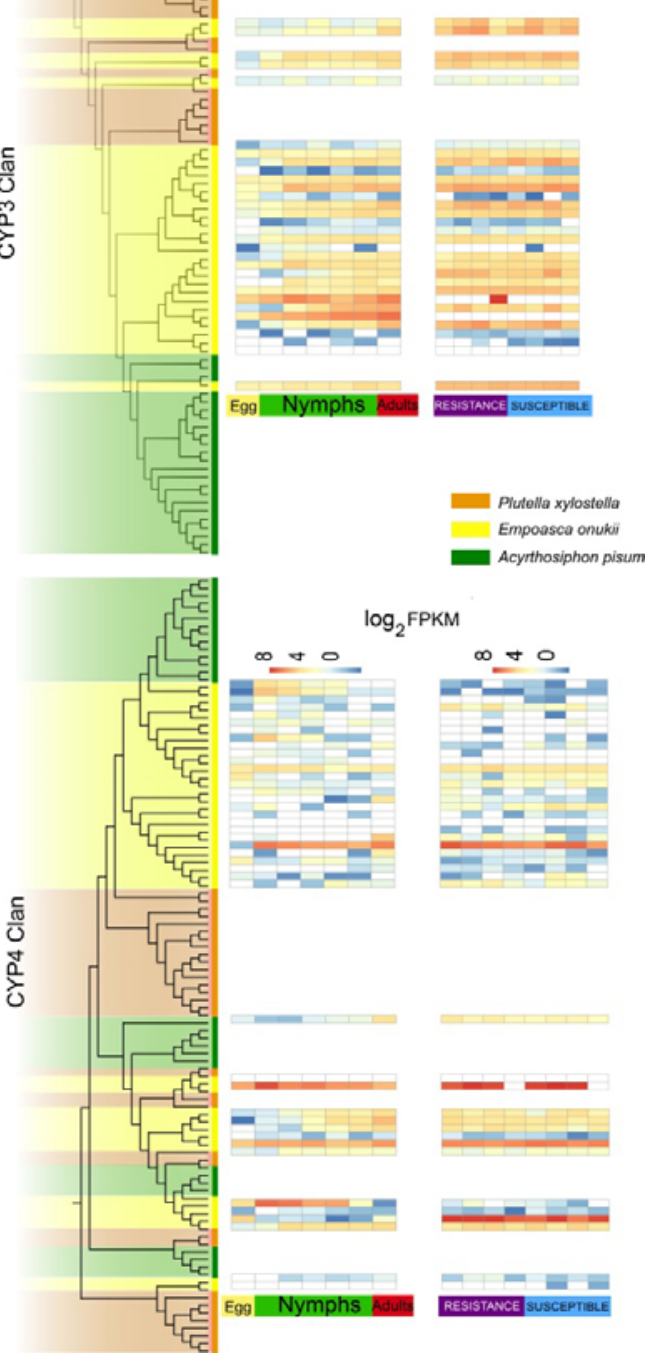

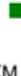

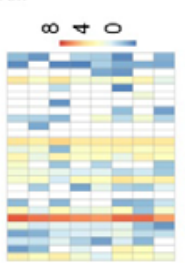

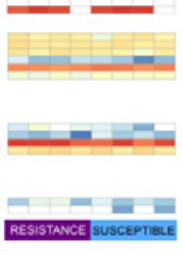

Figure 2. Expansion of gene families related to chemoreception and

detoxification in $E$. onukii compared with other insect species.

(A) Numerical comparison of the chemosensory proteins (CSPs) among aphids, plant bugs and hoppers. Aphid species include Acyrthosiphon pisum (Apis), Myzus persicae (Mper), Aphis gossypii (Agos), Aphis glycines (Agly) and Sitobion avenae (Save); Plant bugs include Adelphocoris lineolatus (Alin), Adelphocoris suturalis (Asut), Apolygus lucorum (Aluc); Hoppers includes Empoasca onukii (Eonu), Laodelphax striatellus (Lstr), Nilaparvata lugens (Nlug) and Sogatella furcifera (Sfur). (B) Phylogenetic relationships of CSPs in hoppers (N. lugens, S. furcifera, and $L$. striatellus), aphids (A. pisum, M. persicae, A. gossypii, A. glycines, and S. avenae), 
and plant bugs (N. lugens, L. striatellus and S. furcifera). Yellow branches represent CSP family genes in E. onukii. (C) Genomic expansion and unbalanced chromosomal distribution of CSPs in the E. onukii genome. (D) Phylogenetic relationships and expression profiling of detoxification-related proteins (CYP3 and CYP4) in plant hoppers, aphids and plant bugs. Expression profiling based on RNA-seq data were generated from all developmental stages (egg, 1st - 5th nymph instar and adult) and 11 E. onukii populations collected from different tea cultivars (four cultivars being resistant including LongJ, DeQ, JianD, JuY and four cultivars susceptible to E. onukii including ZhuS, LanT, BanZ and EnB).
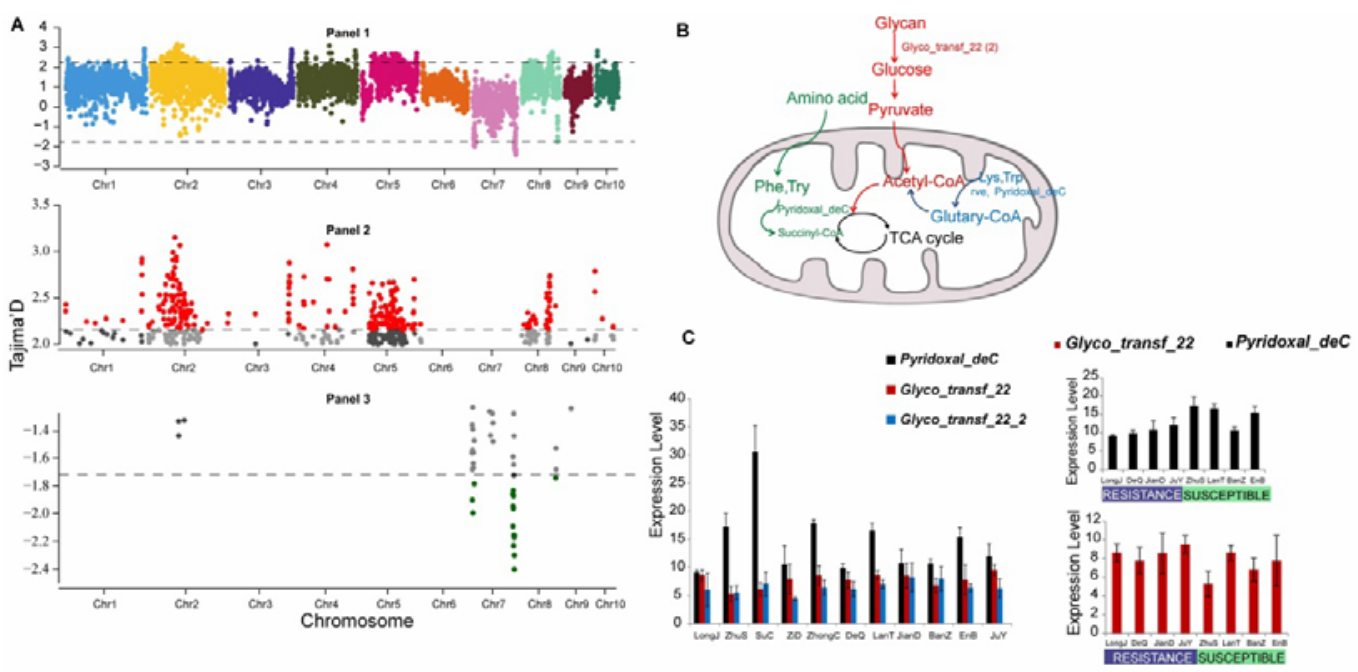

Figure 3. Genomic signatures of balancing selection.

(A) Putative selection sweeps in populations of E. onukii. Tajima's $D$ value was calculated for each of the E. onukii populations. Mean values of Tajima's $D$ are shown in sliding windows of $50 \mathrm{~kb}$ with a step size of $10 \mathrm{~kb}$. Regions with Tajima's $D$ values deviated significantly from 0 are marked with dotted lines in panel 1. Specifically, values of Tajima's $D$ significantly deviated from 0 are plotted in red $(>0)$ and green (<0) respectively in panel 2 and panel 3. (B) Succinyl- and glutaryl-CoA pathways showing the regulatory role of lysine modifications in metabolism. (C) Expression patterns of the E. onukii genes under balancing selection, in 11 different tea cultivars. Pyridoxal_deC was detected to be significantly highly expressed in susceptible tea 
48

49

50

cultivars $(P<0.01$, T-test), while Glyco_transf_22 was significantly highly expressed in resistant tea cultivars $(P<0.05$, T-test $)$.

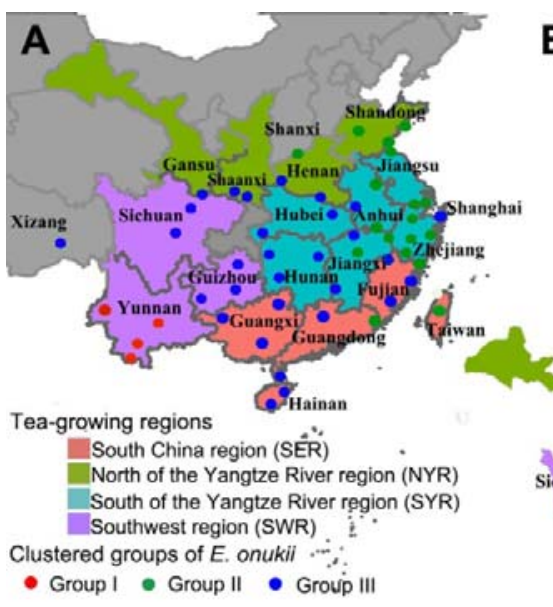

- Group I - Group II - Group III

C Yunnan

Southern-central China
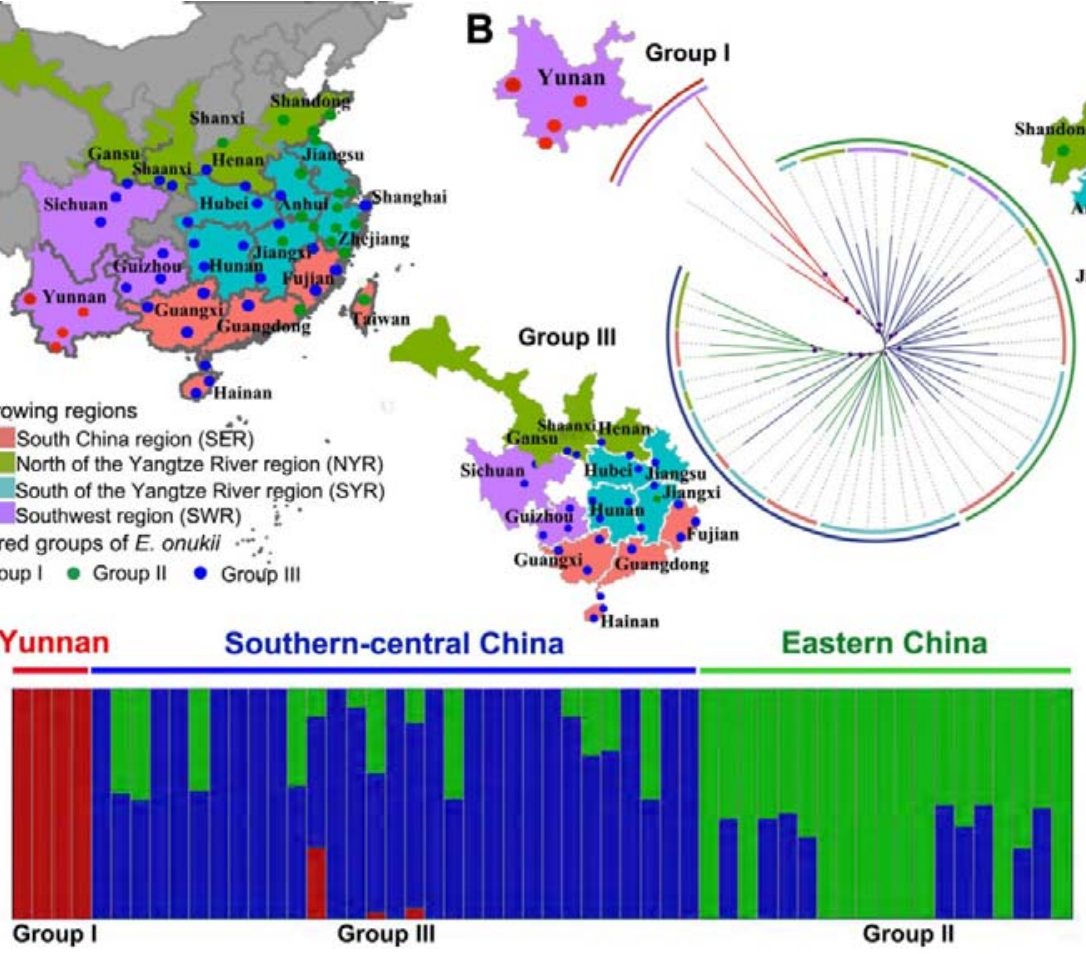

Eastern China

Figure 4. Phylogenetic relationship, population structure and expansion of $E$. onukii.

(A) Geographical locations (sites) of 54 samples collected from four tea-growing regions around China: Southwest region (SWR), South of the Yangtze River region (SYR), North of the Yangtze River region (NYR), and South China region (SER). Dots with different colors represent different clustered groups. (B) Phylogenetic tree of the 54 E. onukii samples based on RAxML and SplitsTress. Branch lengths are not scaled. Different colors of inner circle represent 4 different tea-growing regions shown in Figure 2A. Colors of outer lane represent different E. onukii groups based on phylogenetic analysis. (C) Genetic structure and individual ancestry with colors in each column representing ancestry proportion over range of population sizes $(K=2-4$, with an optimal $K=3$ ). 
Table 1. Sequencing, chromosome-scale assembly and annotation of the $E$. onukii genome

\begin{tabular}{cc}
\hline \multicolumn{2}{c}{ Sequencing } \\
\hline Sequencing Platform & Nanopore ONT \\
Data size $(\mathrm{Gb})$ & 65 \\
Genome sequencing depth $(\times)$ & 109 \\
Estimated genome size (Mb) & $\sim 608$ \\
\hline Chromosome-scale assembly & \\
Assembly size (Mbp) & 599 \\
\% of estimated genome size & 98.5 \\
No. of contigs & 1800 \\
Contig N50 (Mb) & 2.2 \\
Average length (bp) & 332,835 \\
Minimum contig length (bp) & 2,552 \\
No. of chromosomes & 10 \\
Scaffold N50 (Mb) & 67.98 \\
No. of unanchored contigs & 234 \\
Length of anchored contigs (Mb) & 592 \\
Anchor rate (\%) & 98.83 \\
BUSCO completeness (\%) & 92.7 \\
\hline Annotation & 19,642 \\
No. of protein-coding genes & 7904 \\
Average gene length (bp) & 201 \\
Average CDS length (bp) & 4.99 \\
Average exon number per gene & 92.5 \\
BUSCO completeness (\%) & \\
\hline
\end{tabular}


Table 2. Chromosome-based statistics of the $E$. onukii genome

\begin{tabular}{ccc}
\hline Chromosome & No. of contigs & Length (bp) \\
\hline Chr1 & 263 & $94,216,414$ \\
Chr2 & 314 & $91,501,843$ \\
Chr3 & 121 & $74,646,503$ \\
Chr5 & 202 & $67,983,564$ \\
Chr4 & 155 & $65,611,511$ \\
Chr6 & 79 & $54,167,312$ \\
Chr8 & 177 & $48,146,360$ \\
Chr7 & 88 & $43,255,712$ \\
Chr9 & 110 & $28,073,217$ \\
Chr10 & 57 & $24,627,475$ \\
\hline Total number of contigs & & 1800 \\
Total length of contigs (bp) & & $599,103,029$ \\
Total number of anchored contigs & 1566 & \\
Total length of chromosome level assembly (bp) & $592,229,911$ \\
Number of unanchored contigs & 234 \\
Length of unanchored contigs & & \\
Anchor rate (\%) & & $98.028,718$ \\
\hline
\end{tabular}


Table 3. Numerical comparison of genes related to chemoreception and detoxification among different insect species

\begin{tabular}{|c|c|c|c|c|c|c|c|c|c|}
\hline \multirow[b]{2}{*}{ Insect species } & \multicolumn{4}{|c|}{ Chemoreception } & \multicolumn{5}{|c|}{ Detoxification } \\
\hline & Or & $\mathbf{G r}$ & Ir & OBP & CSP & COE & $\mathbf{A B C}$ & GST & P450 \\
\hline A. pisum & 79 & 77 & 11 & 15 & 11 & 57 & 187 & 22 & 83 \\
\hline N. lugens & 50 & 10 & 25 & 11 & 17 & 79 & 40 & 11 & 67 \\
\hline E. onukii & 20 & 12 & 23 & 5 & 26 & 77 & 29 & 30 & 103 \\
\hline P. americana & 154 & 522 & 640 & 6 & 11 & 90 & 115 & 39 & 178 \\
\hline A. mellifera & 163 & 10 & 9 & 21 & 6 & 24 & 41 & 11 & 46 \\
\hline T. castaneum & 299 & 220 & 23 & 49 & 20 & 47 & 73 & 36 & 131 \\
\hline A. gambiae & 79 & 76 & 55 & 82 & 8 & 40 & 55 & 28 & 105 \\
\hline A. aegypti & 131 & 79 & 135 & 111 & 43 & 49 & 69 & 26 & 160 \\
\hline D. melanogaster & 62 & 68 & 66 & 51 & 4 & 35 & 56 & 38 & 85 \\
\hline B. mori & 73 & 76 & 24 & 44 & 20 & 76 & 53 & 20 & 81 \\
\hline P. xylostella & 83 & 26 & 49 & 64 & 20 & 62 & 82 & 22 & 85 \\
\hline
\end{tabular}


Table 4. Number of populations, nuclear SNPs, and genetic diversity $(\pi)$ in each of the three clustered groups

\begin{tabular}{ccccc}
\hline Group & No. populations & No. SNPs & No. Indels & $\boldsymbol{\pi}$ \\
\hline Group I & 4 & $52,089,001$ & $14,913,280$ & 0.004062 \\
Group II & 28 & $472,824,888$ & $135,096,045$ & 0.004744 \\
Group III & 22 & $279,181,783$ & $79,642,551$ & 0.004662 \\
\hline
\end{tabular}

